\title{
TUBERCULOSIS AND LEPROSY: MUTUALLY ANTAGONISTIC DISEASES.*
}

\author{
R. Chaussinand, m.D.
}

"Premunition" to leprosy produced by B.C.G. Vaccine.

Tuberculosis and leprosy are two infections which show numerous points in common. Before the discovery of Hansen's bacillus, certain workers, of whom Danielsen was one, had more or less openly expressed the idea that the two infections were really one. Of course there is no doubt that the two infections are quite separate; nevertheless they present so many points in common that they can be regarded as related diseases.

*(Translation (by J. Lowe) of pp. 146-152 of "La Lèpre" by R. Chaussinand, Head of the Leprosy Department of the Pasteur Institute, Paris, and now Secretary of the Leprosy Expert Panel of the World Health Organization.

"La Lèpre" was published in 1950 by L'Expansion Scientifique Française. The views expressed in the section here translated had been outlined in at least two previous articles. 
Since tuberculosis and leprosy are closely allied, the question has arisen whether there might not occur a certain degree of crossimmunity or premunition pertaining to these two infections.

By studying I,600 cases of leprosy, we satisfied ourselves that a positive Mitsuda reaction to lepromin indicated a condition of relative immunity to leprosy, which showed itself by a marked resistance of the tissues to invasion by the bacilli. Further studies of persons not exposed to leprous infection have shown that such a person infected with tuberculosis, or given B.C.G. vaccine, generally reacts to lepromin; while such a person never infected with tuberculosis and never given B.C.G. vaccine, showed no reaction to lepromin.

These observations recorded in non-leprous persons have been supported by our experiments in animals. Forty guineapigs, Io rabbits, 6 monkeys, and 2 dogs found tuberculin-negative were also found lepromin-negative. In a similar number of similar animals either infected with Koch's bacillus, or vaccinated with B.C.G. the lepromin reaction was positive.

We have further shown that the normal monkey and the guineapig, which are never lepromin-positive, become lepromin-positive after a local leprous infection has been produced by the implant of a piece of leprous nodule.

These findings have led us to the conclusion that immunity to leprosy consists in a state of relative premunition to leprosy, which is the result of a previous infection, either leprous or tuberculous. Since the body infected with tuberculosis appeared to enjoy a certain degree of premunition to leprosy, we considered whether the con. verse might not be true, that is to say, whether the presence of leprous infection in the human body conferred a degree of relative immunity to tuberculosis.

Rogers and Muir, basing their views on the fact frequently recorded that in advanced leprosy, tuberculosis is a common cause of death, consider that leprosy produces no immunity whatever to tuberculosis. This statement appears to us far too dogmatic. We must always keep in mind the fact that leprosy develops in two markedly contrasting forms, the benign, allergic (lepromin-positive) form and the malignant, anergic (lepromin-negative) form. Further we have found that in allergic leprosy, even in the absence of tuberculosis infection, the intradermic injection of an antigen consisting of tubercle bacilli killed by heat produces a positive reaction; the same antigen injected into healthy persons or into persons with anergic (lepromatous) leprosy produces no reaction. Animal experiments have given identical results. The normal guineapig and monkey show no local reaction to Koch's bacillus killed by heat and 
injected locally. Nevertheless, Io guineapigs and 3 monkeys, in which sensitivity to lepromin had been induced by the insertion in the tissues of a fragment of a leprous nodule, were found to have acquired the power to produce a local reaction to the intradermic injection of such killed tubercle bacilli, although the tuberculin reaction was still found negative.

To summarize; a certain degree of resistance to the tubercle bacillus (bacteria para-allergy) is found in allergic leprosy only. It therefore appears to us that it is only to be expected that persons with anergic (lepromatous) leprosy will not show any protection against tuberculosis infection which can be attributed to their leprosy. On the other hand in allergic (tuberculoid) leprosy, we believe we can postulate a state of relative premunition against the tubercle bacillus; this view is based on (a) biological findings above described and (b) on clinical observations.

In general, tuberculosis constitutes a fatal complication of leprosy only in anergic (lepromatous) cases. Allergic (tuberculoid). cases of leprosy usually manage to overcome the primary tuberculous infection, which shows itself most often only by tuberculin sensitivity or sometimes by cervical gland tuberculosis.

Of over 500 cases of allergic (tuberculoid) leprosy examined, we found only 3 cases of progressive pulmonary tuberculosis with tubercle bacilli in the sputum (as shown by culture or guineapig inoculation). In these cases the clinical and bacteriological evidence of active tuberculosis disappeared within a few months of the institution of an artificial pneumothorax. It appears from these observations that cases of allergic leprosy possess a certain degree of resistance to tuberculosis infection.

Tuberculosis and leprosy are thus mutually antagonistic diseases because one can show that there can exist a state of relative cross immunity, produced by these two infections.

Both leprosy and tuberculosis are chronic infectious diseases. Tuberculosis in man is found to be much more infectious and virulent than leprosy. In any population where these two organisms find themselves in competition, the tubercle bacillus spreads more rapidly and widely. And since a previous infection with the tubercle bacillus produces a certain degree of immunity to leprosy, those persons who have been infected with the tubercle bacillus show a state of relative premunition against a later attack by the leprosy bacillus. The gradual but progressive driving out of leprosy will be the predominant feature, and this will be due to the relative cross premunition produced by these two infections.

The truth of our theory of the driving out of leprosy by tuber- 
culosis can be established only by extensive research carried out on the one hand in areas where the spread of leprosy is relatively recent or very marked, and on the other hand in areas where leprosy has declined. The tuberculin-positive rate of the population should be low in regions where leprosy has recently spread. It should be high in regions where it has disappeared. Unfortunately we cannot now produce statistics to prove our theory. All workers on tuberculosis have recognised that the best available statistics are incomplete, and for leprosy, accurate statistics can be considered as non-existent.

In I925, Rogers thought that a population with a high incidence of tuberculosis infection might enjoy a certain degree of immunity to leprosy. This hypothesis however, based only on epidemiological findings concerning leprosy and tuberculosis, was not supported by experimental work.

Behr visited European and Asiatic countries where leprosy is found, and was able to show that there is little tuberculosis in countries where leprosy is endemic, and, conversely, that regions with widespread tuberculous infection were free from leprosy. $\mathrm{He}$ concluded that tuberculous infection does not favour the spread of leprosy.

The spread of leprosy apparently precedes that of tuberculosis, for tuberculosis has not yet attained its peak incidence in most countries of the world, whereas leprosy is markedly declining in many countries. The epidemiological curve of the world incidence of tuberculosis is still in the ascending phase; while that of leprosy is in the declining phase. Moreover it appears that the rise in the curve for tuberculosis has caused, after a longer or shorter interval, the fall of the curve for leprosy.

England and Germany are the two European countries which first attained the peak of the curve of the incidence of tuberculosis (Burnet).

The tuberculosis curve for Norway was still rising in 1890 (Burnet).

The Japanese tuberculosis curve is at its peak, and should soon begin to fall; it has been less broad than the curve in European countries. It rose later and more quickly, and came later under the influence of civilization (Burnet). Moreover the tuberculin-positive rate is relatively low in rural areas.
England and Germany were the first countries in Europe in which leprosy disappeared (Rogers and Muir).

Leprosy has persisted in Norway until the present time. But in fact it is now markedly declining; in I856 2,850 cases compared with i6 in 1948 (Melsom).

Leprosy is still endemic in rural areas of Japan. 
In India, the tuberculosis rate is still rising. The rate is low in rural areas. The people have a poor resistance to tuberculous infection, and the disease frequently takes a rapid course. (Lowe).

In Indo-China the incidence of tuberculosis has not yet reached its peak. The tuberculin positive rate in the town of Saigon-Cholon is about $60 \%$ for children of IO-I 5 years and $80 \%$ in adults. In the provinces a rate of $20 \%$ has been recorded for children of Io years, and $29 \%$ for children I0-17 years.

In Oceania tuberculosis is of relatively recent importation. Ziemann recorded that in I909-I9IO of 4,177 persons hospitalised in Nauru only five showed pulmonary tuberculosis.

In West and Central Africa tuberculosis is rare. The tribes without European contacts are practically free (Calmette).

Civilization by the development of industries, commerce and means of communication spreads tuberculosis.

Tuberculosis spreads first in the towns and later in the countryside.
In India the leprosy rate is probably beginning to decline. The people show a considerable degree of resistance to leprosy as shown by the predominance of the mild forms of the disease in many areas. (Lowe),

In Indo-China leprosy is widespread. The people show a degree of resistance to leprosy and the benign form is more frequent than the malignant form.

In Oceania leprosy is very common. In 1920 following the influenza epidemic, a very high incidence of leprosy was recorded in Nauru (Bray).

West and Central Africa shows foci of leprosy which are the most active in the world (Rogers and Muir).

Civilization in spite of the development of industries, commerce and communications, makes leprosy disappear.

Leprosy declines first in the towns but can remain endemic for long periods in rural areas.

It follows from what has been written above that the progressive driving out of leprosy by tuberculosis constitutes the dominant phenomenon attributable to the relative cross immunity between these two infections.

Further, since B.C.G. vaccination establishes, as do primary infections with leprosy and tuberculosis, a state of allergy to the leprosy bacillus, it seems logical to utilize B.C.G. vaccine in the prophylaxis of leprosy.

In my opinion, the production of premunition by B.C.G. vaccine, which is not difficult, and can be widely applied, deserves to be widely used in areas where leprosy is common, where the fight against leprosy presents so many difficulties, and where the future extension of tuberculosis constitutes such a terrible menace. 